\title{
Densidade Crítica no Modelo de Percolação em Rede de Bethe Não-Homogênea
}

\author{
H.R. TAVARES ${ }^{1 *}$, R.N.C. SOUZA ${ }^{2}$, M.R. MADRUGA ${ }^{1}$ e V.J.C. FARIAS ${ }^{1}$ \\ Recebido em 14 agosto, 2014 / Aceito em 26 julho, 2015
}

\begin{abstract}
RESUMO. Neste trabalho tratamos de um modelo percolação não-homogênea na rede de Bethe cuja probabilidade de um elo no nível $n$ estar aberto muda de acordo $\operatorname{com} n$. Este modelo pode ser apropriado para situações onde o meio muda sua densidade de forma sistemática, tal como a proliferação de insetos que dependem da temperatura e umidade, que variam entre dia e noite. Consideramos o caso onde a probabilidade de um elo $e_{n}$ do nível $n$ estar aberto é dada pela função senóide $\bar{p}\left(e_{n}\right)=p+(1-p)|\operatorname{sen}(n)|$. Para este modelo apresentamos resultados de simulações Monte-Carlo que indicam um comportamento da função de percolação com transição de fase de segunda ordem em $p_{c}$, mas provamos analiticamente a existência de um ponto crítico não trivial, apresentando a expressão para a obtenção desta probabilidade crítica.
\end{abstract}

Palavras-chave: percolação não-homogênea, rede de Bethe, ponto crítico.

\section{INTRODUÇÃO}

A Teoria da Percolação trata do fenômeno da propagação de um fluido em meio poroso, seja água ou gás no interior de uma rocha. Broadbent \& Hammersley (1957) propuseram o primeiro modelo deste fenômeno. Desde então, muitos outros modelos têm sido propostos para representar o meio poroso. Nos últimos anos, grande progresso tem sido alcançado relativamente às técnicas usadas para resolver problemas de percolação, e também substancial expansão dos modelos de percolação e suas aplicações em várias áreas do conhecimento científico, tais como física, química, biologia, geologia, engenharia, e muitas outras. Existem modelos que consideram a expansão de incêndios florestais, enquanto outros consideram o crescimento de tumores em organismos vivos ou condução de eletricidade em materiais semicondutores. Usualmente o modelo considera o sistema como um meio homogêneo, de forma que a medida de probabilidade $p$ é constante para todos os elos da rede. Porém, no mundo real existem vários sistemas não totalmente homogêneos, havendo uma componente sistemática tal que o modelo ideal pode ser

\footnotetext{
*Autor correspondente: Heliton R. Tavares

${ }^{1}$ Faculdade de Estatística/PPGME, UFPA, Universidade Federal do Pará, 66075-110 Belém, PA, Brasil.

E-mails: heliton@ufpa.br; madruga@ufpa.br; valcir@ufpa.br

2 Programa de Pós-Graduação em Matemática e Estatística, UFPA, Universidade Federal do Pará, 66075-110 Belém, PA, Brasil. E-mail: nonato-de-souza@hotmail.com
} 
baseado em um sistema composto não uniforme de probabilidade para todo meio. A probabilidade $\bar{p}($.) pode variar em função do espaço ou do tempo, por exemplo.

A rede de Bethe ou árvore Cayley foi introduzida em Bethe (1935) no contexto da mecânica estatística. Ela pode ser usada para fazer aproximação de modelos de percolação em grandes dimensões. Em geral, a rede de Bethe é definida como um grafo $\mathbb{G}=(\mathbb{V}, \mathbb{E})$, onde $\mathbb{V}$ é um conjunto de pontos, também chamados vértices ou sítios, e $\mathbb{E}=\left(e_{x y}\right)$ é o conjunto não ordenado de pares de vértices distintos $e_{x y}$, chamados elos ou arestas (veja Braga et al. (2005), por exemplo). Então, a rede de Bethe é um grafo onde qualquer vértice está conectado com $r$ outros, onde $r$ é chamado de número de coordenação. Vamos denotar por $B_{r}$ a rede de Bethe com número de coordenação $r$ (veja a Figura 1 para ilustração de $B_{3}$ ). Na Figura 1(a) pode ser visto a estrutura das árvores que emanam de um vértice central, e todos os vértices estão organizados em níveis sequenciais em torno de um vértice central. $O$ vértice central pode ser chamado de raiz ou origem da rede. A Figura 1(b) mostra uma representação alternativa similar à água saindo de uma fonte.

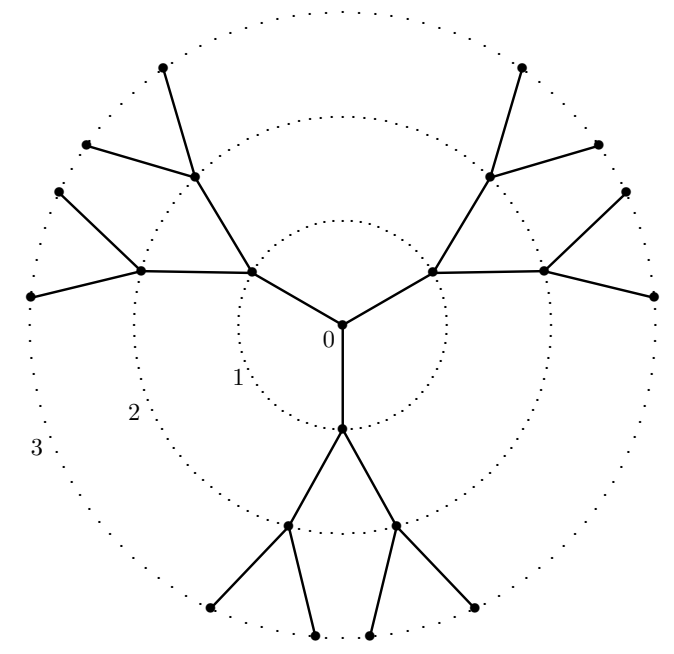

(a) Representação circular

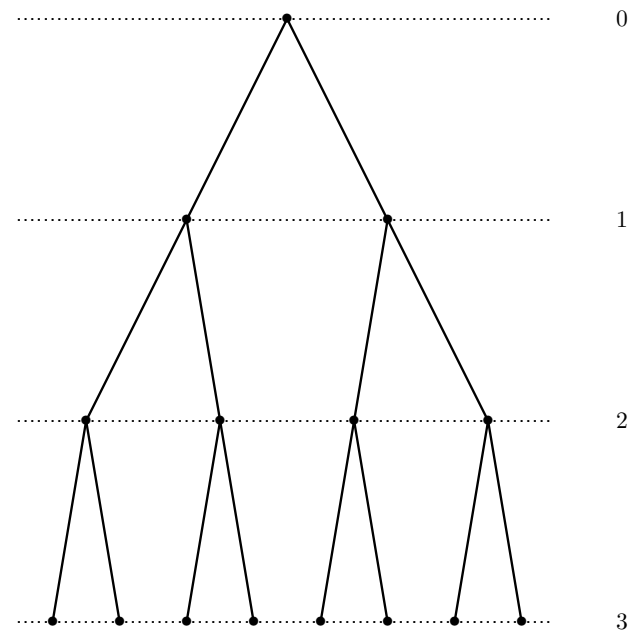

(b) Representação alternativa

Figura 1: Rede de Bethe.

Em $B_{r}$ a distância de qualquer vértice $x$ até a origem é conhecida como nível do vértice e é denotada por $l(x)$. Sendo $e_{x}$ um elo qualquer finalizado em $x$, diremos que este pertence ao nível $l(x)$. Por simplicidade de notação, vamos representar por $e_{n}$ todos os elos $e_{x}$ tais que $l(x)=n$.

É usual considerar o subgrafo $B_{r}(n)$ de $B_{r}$, formado pelos vértices $x$ tal que $l(x) \leq n$ e pelos elos conectados a estes vértices. O conjunto $B_{r}(n)$ é conhecido por ser a rede de Bethe com "volume finito". A fronteira de $B_{r}(n)$, denotada por $\partial B_{r}(n)$, é o conjunto de todos os vértices do nível $n$, e podemos ver que o número de vértices até $n$ é igual a $\left|\partial B_{r}(n)\right|=r(r-1)^{n-1}$ pela representação da Figura 1(a), e igual a $(r-1)^{n}$ pela representação da Figura 1(b). 
Na rede de Bethe homogênea, a cada elo $e \in E$ associamos uma variável aleatória independente $X_{e}$ com distribuição de Bernoulli com parâmetro $p$ que chamamos de medida de probabilidade. Se $X_{e}=1$ diremos que $e$ está aberto, caso contrário $e$ está fechado. Para $x, y \in B_{r}$, diremos que $x$ está conectado a $y$ e denotamos por $x \stackrel{p}{\leftrightarrow} y$ se existir uma sequência de vértices distintos $x=x_{0}, x_{1}, \cdots, x_{n}=y$, tais que os elos $e_{x_{i-1}, x_{i}}, i=1, \cdots, n$, estejam abertos. A função de conectividade, denotada por $\tau_{x y}(p)$, é a probabilidade dos vértices $x$ e $y$ estarem conectados por um caminho de elos abertos, i.e., $\tau_{x y}(p)=P(\{x \stackrel{p}{\leftrightarrow} y\})$. Podemos mostrar que a função conectividade em $B_{r}$ decresce exponencialmente quando $\|x-y\| \rightarrow \infty$ para todo $p<1$ (Braga et al., 2005). Isto pode ser facilmente visto, pois $\tau_{x y}(p)=\exp (-\|x-y\| / \zeta(p))$, com $\zeta(p)=(-\log (p))^{-1}$. A função $\zeta$ é usualmente referida como comprimento de correlação. Portanto, em uma rede de Bethe, o comprimento de correlação é finito para todo $0<p<1$.

O Aglomerado da Origem (O) é representado por $C(p)=\left\{x \in B_{r}: \mathbf{O} \stackrel{p}{\leftrightarrow} x\right\}$ e a função probabilidade de percolação é dada por $\theta(p)=P_{p}(|C(p)|=\infty)$, onde $|C(p)|$ representa o número de vértices em $C(p)$. Diremos que ocorreu percolação se $|C(p)|=\infty$.

Outra importante definição é a esperança matemática do aglomerado, também conhecida como susceptibilidade, dada por $\chi(p)=E(C(p))=\sum_{x \in B_{r}} \tau_{0 x}(p)$. Associado a $\chi(p)$ e $\theta(p)$ há expoentes críticos, $\gamma$ e $\beta$, tais que $\chi(p) \approx\left(p-p_{c}\right)^{\gamma}$ quando $p \uparrow p_{c}$, e $\theta(p) \approx\left(p-p_{c}\right)^{\beta}$ quando $p \downarrow p_{c}$, no sentido que $-\log \chi(p) / \log \left(p-p_{c}\right) \rightarrow \gamma \mathrm{e}-\log \theta(p) / \log \left(p-p_{c}\right) \rightarrow \beta$.

\section{RESULTADOS IMPORTANTES NA REDE DE BETHE}

O caso $r=2$ não é teoricamente interessante, pois se $p<1$ teremos uma infinidade de elos fechados à direita e à esquerda da origem, tais que $\theta(p)=0$ se $p<1$. Então, concluímos que $p_{c}=1$, e não ocorre a transição de fase. Para $r \geq 3$ pode ser provado que existe um $p_{c} \in(0,1)$, tal que $\theta(p)=0$ para $p<p_{c}$ e $\theta(p)>0$ para $p>p_{c}$. A forma da função $\theta$ para $p>p_{c}$ pode ser obtida implicitamente por $1-\theta(p)=(1-p \theta(p))^{r-1}$. Para $r=3$ temos que $\theta(p)=1-[(1-p) / p]^{2}$ e para $r=4, \theta(p)=(3-\sqrt{4 / p-3}) /(2 p)$, por exemplo. Sabemos que $p_{c}=1 /(r-1)$ e que $\theta\left(p_{c}\right)=0$. Neste sentido, seja $S_{r}^{\bullet}(n, p)$ uma variável aleatória representando o número de vértices no nível $n \geq 1$ com caminho de elos abertos até a origem, com $S_{r}^{\bullet}(0, p) \equiv 1$. Temos que a distribuição de $S_{r}^{\bullet}(n, p) \mid\left\{S_{r}^{\bullet}(n-1, p)=x\right\}$ é $\operatorname{Binomial}(x(r-1), p)$ e sua esperança é $\mathbb{E}\left(S_{r}^{\bullet}(n, p) \mid\left[S_{r}^{\bullet}(n-1, p)\right]=S_{r}^{\bullet}(n-1, p)(r-1) p\right.$. Com isto, temos que

$$
\mathbb{E}\left(S_{r}^{\bullet}(n, p)\right)=[(r-1) p]^{n} \begin{cases}\rightarrow 0 & \text { para } n \uparrow \infty \text { se } p<\frac{1}{r-1} \\ =1 & \text { se } p=\frac{1}{r-1} \\ \rightarrow \infty & \text { para } n \uparrow \infty \text { se } p>\frac{1}{r-1} .\end{cases}
$$

Notemos que se $\mu$ é o número esperado de elos abertos partindo da origem (ou qualquer outro vértice), temos que $\mu=(r-1) p$. Portanto, se $\mu \leq 1$ o sistema não percola, e se $\mu>1$ teremos probabilidade positiva de percolação. Resultados gerais podem ser obtidos em Galton-Watson branching process (Haccou et al., 2005; Bruss, 1984; Bollobás et al., 2014). 
Se considerarmos $\theta_{n}(p)$ a probabilidade da origem estar conectada à fronteira $\partial B_{r}(n)$, dada por $\theta_{n}(p)=P_{p}\left\{O \stackrel{p}{\leftrightarrow} \partial B_{r}(n)\right\}$, então a função probabilidade de percolação pode se escrita como o limite de $\theta_{n}(p)$ quando $n \rightarrow \infty$. Para $r=3$ no nível $n=1$ temos $\theta_{1}(p)=p(2-p)$; para $n=2$ temos $\theta_{2}(p)=4 p^{2}-2 p^{3}-4 p^{4}+4 p^{5}-p^{6}$. De modo geral, seja

$$
c_{i j}^{(n)}=P\left(S_{r}^{\bullet}(n, p)=j \mid S_{r}^{\bullet}(n-1, p)=i\right) \quad \text { e } \quad u_{j}^{(n)}=P\left(S_{r}^{\bullet}(n, p)=j\right)
$$

as probabilidades condicional e incondicional (abandonaremos $p$ e $r$ para facilitar a notação), e $C^{(n)}=\left(c_{i j}^{(n)}\right)$ é a representação matricial com $\left|\partial B_{r}(n)\right|+1$ linhas e $\left|\partial B_{r}(n-1)\right|+1$ colunas; do mesmo modo, $\boldsymbol{u}^{(n)}=\left(u_{j}^{(n)}\right)_{j \geq 0}$ é o vetor de dimensão $\left|\partial B_{r}(n)\right|+1$. Então, podemos escrever para $n \geq 1$,

$$
\begin{aligned}
\boldsymbol{u}^{(n)} & =\boldsymbol{C}^{(n)} \times \boldsymbol{u}^{(n-1)} \\
& =\boldsymbol{C}^{(n)} \times \boldsymbol{C}^{(n-1)} \times \boldsymbol{C}^{(n-2)} \times \cdots \times \boldsymbol{C}^{(1)} \times \boldsymbol{C}^{(0)} \\
& =\prod_{k=0}^{n} \boldsymbol{C}^{(n-k)}
\end{aligned}
$$

com $\boldsymbol{C}^{(0)}=\boldsymbol{u}^{(0)} \equiv(0,1)^{\prime}$. Para $r=3$ e fazendo $q=1-p$, temos que

$$
\begin{gathered}
\boldsymbol{C}^{(1)}=\left(\begin{array}{cc}
1 & q^{2} \\
0 & 2 p q \\
0 & p^{2}
\end{array}\right), \boldsymbol{C}^{(2)}=\left(\begin{array}{ccc}
1 & q^{2} & q^{4} \\
0 & 2 p q & 4 p q^{3} \\
0 & p^{2} & 6 p^{2} q^{2} \\
0 & 0 & 4 p^{3} q \\
0 & 0 & p^{4}
\end{array}\right) \text { e } \\
\boldsymbol{C}^{(3)}=\left(\begin{array}{ccccc}
1 & q^{2} & q^{4} & q^{6} & q^{8} \\
0 & 2 p q & 4 p q^{3} & 6 p q^{5} & 8 p q^{7} \\
0 & p^{2} & 6 p^{2} q^{2} & 15 p^{2} q^{4} & 28 p^{2} q^{6} \\
0 & 0 & 4 p^{3} q & 20 p^{3} q^{3} & 56 p^{3} q^{5} \\
0 & 0 & p^{4} & 15 p^{4} q^{2} & 70 p^{4} q^{4} \\
0 & 0 & 0 & 6 p^{5} q & 56 p^{5} q^{3} \\
0 & 0 & 0 & p^{6} & 28 p^{6} q^{2} \\
0 & 0 & 0 & 0 & 8 p^{7} q^{1} \\
0 & 0 & 0 & 0 & q^{8}
\end{array}\right) .
\end{gathered}
$$

As colunas de $C^{(n)}$ são o desenvolvimento de $(q+p)^{k}$ em termos binomiais para $k \in\left\{\left|\partial B_{r}(0)\right|\right.$, $\left.\left|\partial B_{r}(1)\right|, \cdots,\left|\partial B_{r}(n)\right|\right\}$. Então, $\boldsymbol{C}^{(n-1)}$ é uma submatriz de $\boldsymbol{C}^{(n)}, n \geq 1$, como podemos ver acima.

O primeiro elemento de $\boldsymbol{u}^{(n)}$ é a probabilidade condicional de não existir nenhum vértice ocupado no nível $n$. Assim, podemos escrever $\theta_{n}(p)=1-u_{0}^{(n)}$.

Podemos notar que para $n$ pequeno $(n>1)$, a função $\theta_{n}$ tem formato tipo $S$ em [0,1], ficando mais íngreme em $p_{c}$ quando $n$ cresce. A Figura 2 mostra o comportamento de $\theta_{n}$ para $r=3$ 
e vários valores de $n$. Obter o limite quando $n \rightarrow \infty$ do ponto de inflexão das curvas $\theta_{n}(p)$ é um dos métodos usados para determinar o ponto crítico do sistema, resolvendo $\partial^{2} \theta_{n}(p) / \partial p^{2}=$ $-\partial^{2} u_{0}^{(n)} / \partial p^{2}=0$ (Vogel et al., 2010).

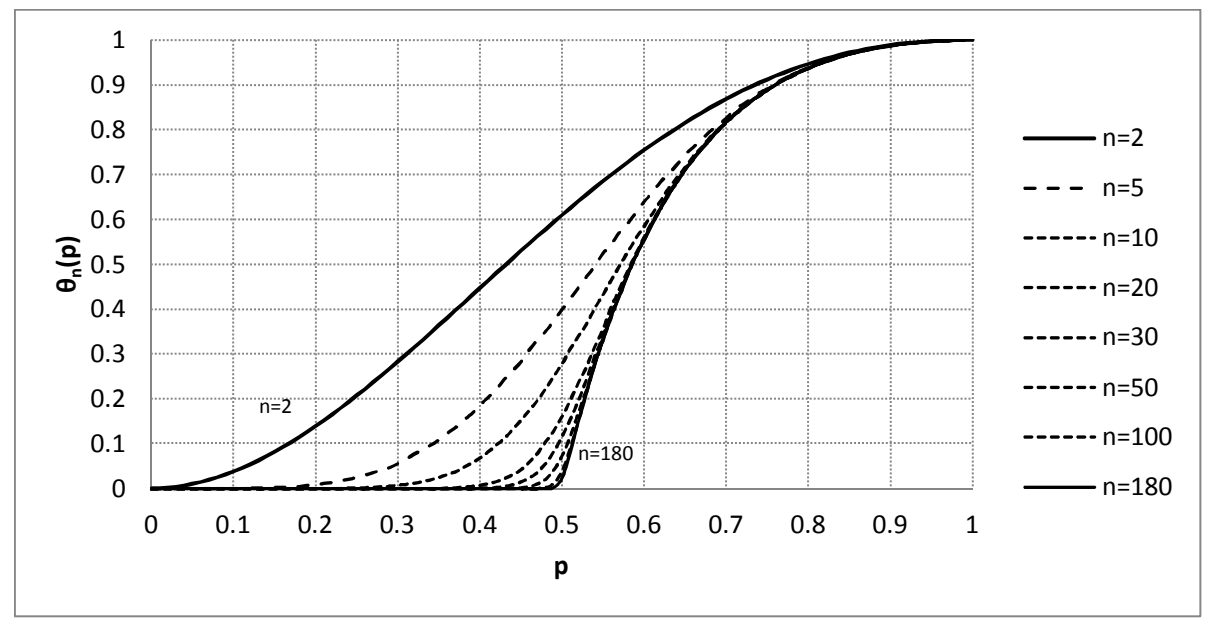

Figura 2: Função probabilidade $\theta_{n}$ para $r=3$.

\section{PERCOLAÇÃO NÃO-HOMOGÊNEA}

Na percolação não-homogênea normalmente supõe-se que elos do mesmo tipo têm a mesma probabilidade de estarem abertos. Por exemplo, na rede quadrada $(\mathbb{S})$ os elos são naturalmente divididos em duas classes, horizontal e vertical, com probabilidades $p_{h}$ e $p_{v}$, respectivamente. Nas redes triangular $(\mathbb{T})$ e hexagonal $(\mathbb{H})$ os elos podem ser descritos em três classes, com probabilidades $p_{0}, p_{1}$ e $p_{2}$. Denotamos a medida de probabilidade associada à rede $\mathbb{S}$ por $\theta_{\mathbb{S}}(\boldsymbol{p})$ onde $\boldsymbol{p}=\left(p_{h}, p_{v}\right)$. Nas redes triangular e hexagonal, a medida é definida por $\boldsymbol{p}=\left(p_{0}, p_{1}, p_{2}\right)$, com funções de probabilidade $\theta^{\mathbb{T}}$ e $\theta^{\mathbb{H}}$. Em qualquer rede, temos agora uma superfície critica $K^{+}($.$) ,$ ao invés de pontos críticos, satisfazendo $K^{+}(\boldsymbol{p})=0$ (veja Grimmett (1999); Kesten (1982) e Grimmett \& Manolescu (2013)):

$$
\begin{aligned}
K^{\mathbb{S}}(\boldsymbol{p}) & =p_{h}+p_{v}-1 \\
K^{\mathbb{T}}(\boldsymbol{p}) & =p_{0}+p_{1}+p_{2}-p_{0} p_{1} p_{2}-1 \\
K^{\mathbb{H}}(\boldsymbol{p}) & =-K^{\mathbb{T}}\left(1-p_{0}, 1-p_{1}, 1-p_{2}\right) .
\end{aligned}
$$

\section{PERCOLAÇÃO EM ONDAS: MODELANDO A PROBABILIDADE NÃO-HOMOGÊNEA $\bar{p}($.)}

A maioria dos modelos de percolação propostos na literatura considera que a probabilidade de um elo qualquer da rede estar aberto é constante para todo meio, ou possui dois ou três possíveis valores de acordo com sua categoria, o que talvez não se aproxime da realidade em alguns casos. Por exemplo, o caso biológico do avanço de tumores em organismos vivos em diferentes 
condições imunológicas ou tipos celulares, ou o caso de um incêndio florestal onde em uma certa região mais úmida, a probabilidade do fogo se espalhar é menor que em outra região mais seca, onde a probabilidade é naturalmente maior. Neste modelo, a altitude, também pode ser considerada importante variável. No estudo da infiltração de fluidos (gasolina, por exemplo) no solo, este pode ter regiões mais compactas, correspondendo à baixa probabilidade, e regiões mais permeáveis, correspondendo à maior probabilidade. Esta probabilidade pode ser modelada em função do tempo ou distância da origem.

Neste sentido, consideremos que um elo $e_{n}$ no nível $n$ da rede de Bethe tem probabilidade de estar aberto dada por

$$
\bar{p}\left(e_{n}\right)=p+(1-p)|\operatorname{sen}(n)| .
$$

Com esta estrutura, que denominaremos por modelo de ondas senóide, precisamos avaliar o comportamento da probabilidade de percolação e a probabilidade crítica em função de $p$, a densidade mínima que a rede pode assumir. O valor numérico de $\bar{p}\left(e_{n}\right)$ será igual a $p$ periodicamente nos níveis $n$ múltiplos de 180 , porque teremos $\operatorname{sen}(n)=0$. Também, $\bar{p}\left(e_{n}\right)$ será periodicamente igual a 1 , nos níveis $n$ tal que $n=90+180 k, k=0,1,2, \ldots$ Em todos os outros elos, $\bar{p}\left(e_{n}\right)$ estará sempre entre $p$ e 1 .

É claro que $\bar{p}(e) \geq p, \forall e \in \mathbb{E}$. Tal como no caso regular, para todo elo $e \in \mathbb{E}$ associamos uma variável aleatória independente $X_{e}^{*}$ com distribuição Bernoulli de parâmetro $\bar{p}(e)$. Por acoplamento, temos que $X_{e}^{*} \geq X_{e}$ e por analogia, $C^{*}, \theta^{*}(p)$ e $p_{c}^{*}$. Temos, portanto, que

$$
C \subset C^{*}, \quad \theta^{*}(p) \geq \theta(p) \text { e } \quad p_{c}^{*} \leq p_{c} .
$$

Antes de obtermos analiticamente as principais característica deste modelo, apresentaremos alguns resultados obtidos por simulações Monte Carlo para estimar a função $\theta^{*}(p)$. Usaremos $M=10^{6}$ réplicas do processo de percolação para vários valores de $p$ de zero até 1 com incremento de 0.001. O nível máximo da rede de Bethe será $n=10^{5}$.

A primeira curva à direita na Figura 3 refere-se à rede de Bethe homogênea com $r=3$, muito próximo da função de probabilidade teórica, mostrando que o algoritmo funciona apropriadamente. As outras curvas referem-se ao modelo de percolação não-homogênea em (4.1), indicando que a função de percolação $\theta^{*}$ comporta-se como uma função tipo $S$, isto é, uma função de distribuição acumulada, característica que não ocorre no modelo homogêneo $[\bar{p}(e)=p]$ para $n$ grande, como podemos ver que na Figura 2. O comportamento da Figura 3 provoca uma importante questão: podemos dizer que $p_{c}^{*}>0$ ? Com o comportamento em $S$, não é trivial afirmar que $p_{c}^{*}>0$, principalmente no caso em que $r \geq 6$. Uma prova formal de que $p_{c}^{*}$ é ou não positivo é necessária, pois é uma das principais características do modelo.

\section{OBTEÇÃO DA DENSIDADE CRÍTICA NÃO TRIVIAL NO MODELO DE ONDAS SENÓIDE}

Nesta seção verificaremos que existe um limiar de percolação não trivial para este sistema, isto é, $p_{c}^{*}>0$. Primeiro vamos encontrar um função similar ao comprimento de correlação que 


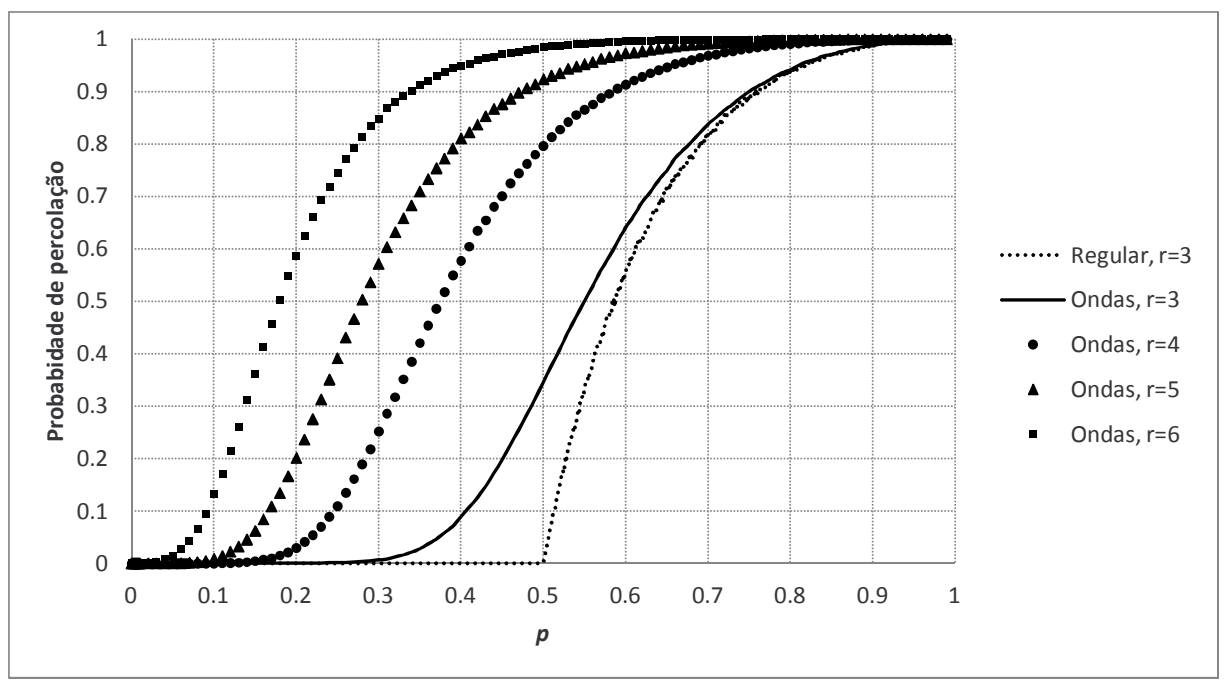

Figura 3: Probabilidade de percolação não-homogênea para $r=3,4,5,6$ e caso homogêneo para $r=3$ com $p_{c}=1 /(r-1)$.

converge para a função conectividade, e que será determinante para a obtenção da probabilidade crítica.

\subsection{A função conectividade e o comprimento de correlação}

A função conectividade da origem até o vértice $x \operatorname{com} l(x)=n$, através do caminho $0, x_{1}, x_{2}, \ldots$, $x_{n}=x$, e respectivos elos $e_{1}, e_{2}, \ldots, e_{n}$, será dada por

$$
\tau_{0 x}(p)=\prod_{k=1}^{n} \bar{p}\left(e_{k}\right) .
$$

Seja $0, x_{1}, x_{2}, \cdots, x_{n}=x$ o caminho de tamanho $n$ da origem até $x$, e $\tau_{0 x}(p)$ a função conectividade. Assim, existe uma função positiva monotônica $\zeta$ tal que, para $n$ grande,

$$
\tau_{0 x}(p) \simeq \exp \{-n / \zeta(p)\} .
$$

Para provar (5.2), notemos que

$$
\begin{aligned}
\tau_{0 x}(p) & =\prod_{k=1}^{n} \bar{p}\left(e_{k}\right)=\exp \left\{\sum_{k=1}^{n} \log \bar{p}\left(e_{k}\right)\right\} \\
& =\exp \left\{n \times \frac{1}{n} \sum_{k=1}^{n} \log \bar{p}\left(e_{k}\right)\right\},
\end{aligned}
$$


em que log é tomado na base neperiana. Seja

$$
\zeta_{n}(p)=\left[-\frac{1}{n} \sum_{k=1}^{n} \log \bar{p}\left(e_{k}\right)\right]^{-1} .
$$

Para encontrar o limite de (5.3), primeiro notemos que $\bar{p}\left(e_{k}\right)$ é cíclico com período $T=180$, com $\operatorname{sen}(k) \geq 0$ para $k \in\{1, \cdots, T\}$ e $\bar{p}\left(e_{k}\right)=1$ quando $p=1$. Assim, as somas consecutivas de $T$ elementos são constantes e, então, $\zeta_{n}(p)$ converge para $\zeta_{T}(p)$ quando $n \rightarrow \infty$, com

$$
\zeta_{T}^{-1}(0)=-\frac{1}{T} \sum_{k=1}^{T} \log (\operatorname{sen}(k)) \geq-\frac{1}{T} \log (\operatorname{sen}(T))=\infty
$$

$\mathrm{e}$

$$
\zeta_{T}^{-1}(1)=-\frac{1}{T} \sum_{k=1}^{T} \log (1)=0
$$

Temos então que $\zeta(p)=\zeta_{T}(p), \operatorname{com} \zeta(0)=0$ e $\zeta(1)=\infty$. Na Figura 4 apresentamos o gráfico de $\zeta(p)$.

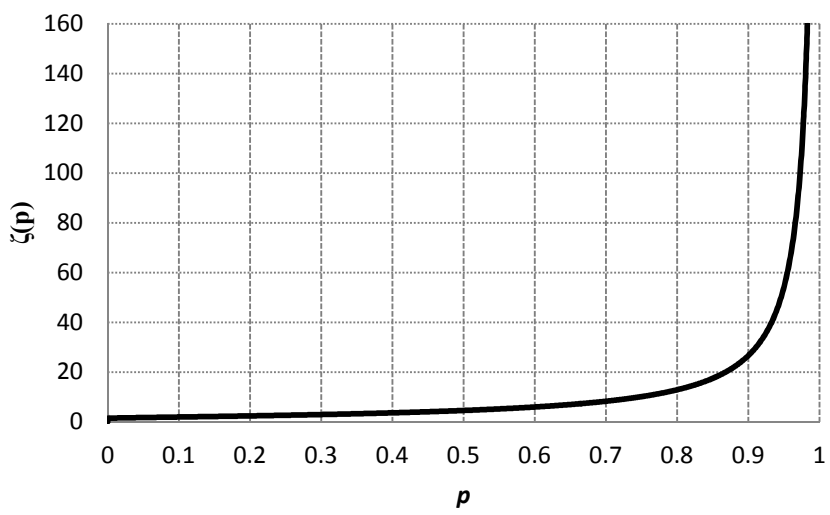

Figura 4: Comportamento de $\zeta(p)$.

\subsection{Densidade crítica}

Para a obtenção do ponto crítico desta rede usaremos um desenvolvimento similar ao de (2.1). Considerando novamente $S_{r}^{\bullet}(n, p)$ uma variável aleatória representando o número de vértices no nível $n \geq 1, \operatorname{com} S_{r}^{\bullet}(0, p) \equiv 1$, temos que

$$
S_{r}^{\bullet}(n, p) \mid\left\{S_{r}^{\bullet}(n-1, p)=x\right\} \sim \operatorname{Binomial}\left(x(r-1), \bar{p}_{n}\right),
$$

onde $\bar{p}_{n}=\bar{p}\left(e_{n}\right)$. A esperança condicional é

$$
\mathbb{E}\left(S_{r}^{\bullet}(n, p) \mid S_{r}^{\bullet}(n-1, p)\right)=S_{r}^{\bullet}(n-1, p)(r-1) \bar{p}_{n}
$$


e a esperança incondicional é obtida recursivamente por

$$
\begin{aligned}
\mathbb{E}\left(S_{r}^{\bullet}(n, p)\right)= & (r-1) \bar{p}_{n} \mathbb{E}\left(S_{r}^{\bullet}(n-1, p)\right) \\
= & (r-1)^{2} \bar{p}_{n} \bar{p}_{n-1} \mathbb{E}\left(S_{r}^{\bullet}(n-2, p)\right) \\
\vdots & \\
= & (r-1)^{n} \prod_{k=1}^{n} \bar{p}_{k} \\
= & (r-1)^{n} \exp \left(-n / \zeta_{n}(p)\right) \\
= & \exp \left\{-n\left[\zeta_{n}^{-1}(p)-\log (r-1)\right]\right\} \\
& \stackrel{n \rightarrow \infty}{=} \begin{cases}0 & \text { se } \zeta(p)<\frac{1}{\log (r-1)} \\
1 & \text { se } \zeta(p)=\frac{1}{\log (r-1)} \\
\infty & \text { se } \zeta(p)>\frac{1}{\log (r-1)} .\end{cases}
\end{aligned}
$$

Então, concluímos que $p_{c}^{*}$ é dado pela solução de

$$
\zeta(p)=\frac{1}{\log (r-1)}
$$

O comprimento de correlação é uma função continua de $p$, assim podemos obter com alta precisão o valor do ponto crítico. A solução numérica para $r=3$ é $p_{c}^{*}=0.00148254$, e para $r=4$ temos $p_{c}^{*}=0.558815 \times 10^{-34}$. É importante notar que (5.4) é verdade também para o caso regular $(\bar{p}(e)=p)$, onde temos $\zeta(p)=(-\log (p))^{-1}$ e a solução de (5.4) é dada por $p_{c}=1 /(r-1)$.

\section{CONCLUSÕES}

Apresentamos um modelo de percolação de rede de Bethe não-homogênea considerando a probabilidade de um elo estar aberto em função da distância $l(e)$ deste até a origem, dada por uma função senóide $\bar{p}(e)=p+(1-p)|\operatorname{sen}(l(e))|$. Não existe na literatura nenhum modelo periódico similar usado em percolação em outros contextos. Um estudo de simulação preliminar indicou que a função probabilidade de percolação não é uma função abrupta, diferente do caso regular (homogêneo), caracterizando uma transição de fase de segunda ordem e tornando imprecisa a conclusão sobre se o limiar de percolação é ou não trivial (zero). Demonstramos que a probabilidade de percolação é estritamente positiva. Um programa de simulação feito pelos autores mostra o comportamento da probabilidade de percolação baseados em $M=10^{6}$ réplicas do processo percolação para $p$ variando de zero até 1 com incremento 0.001 , com nível máximo da rede de $n=10^{5}$. É importante notar que várias extensões deste modelo podem ser feitas (van den Berg et al., 2012; Chalupa et al., 1979) para casos parecidos ou outras situações mais realistas. 


\begin{abstract}
In this work we deal with an inhomogeneous Bethe lattice percolation model where the probability of an edge in level $n$ is open changes according as $n$. This model can be appropriate to the case where the media changes its density in a systematic way, such as the proliferation of insects that depends on the temperature and humidity, which fluctuates between day and night. We consider the case where the density $\bar{p}($.$) follows a function of the$ distance $l($.) from the origin, given by a sinusoid function $\bar{p}()=.p+(1-p)|\sin (l())$.$| . For$ this model we present results of Monte-Carlo simulation showing the behavior of the probability of percolation with a second-order phase transition, but we present too a formal proof that the density is non trivial, with the mathematical expression to compute the percolation threshold.
\end{abstract}

Keywords: inhomogeneus percolation, Bethe lattice, critical point.

\title{
REFERÊNCIAS
}

[1] H.A. Bethe. Statistical theory of superlattices. Proc. Roy. Soc. London, Ser. A, 150 (1935), 552-575.

[2] B. Bollobás, K. Gundersony, C. Holmgrenz, S. Jansonx \& M. Przykucki. Bootstrap percolation on Galton-Watson trees. Electron. J. Probab., 19(13) (2014), 1-27.

[3] G.A. Braga, R. Sanchis \& T. A. Schieber. Critical Percolation on a Bethe Lattice Revisited. SIAM REVIEW, 47(2) (2005), 349-365.

[4] S.R. Broadbent \& J.M. Hammersley. Percolation process I. Crystals ans mazes. Proceedings of the Cambridge Philosofical Society, 53 (1957), 629-641.

[5] F.T. Bruss. A Note on Extinction Criteria for Bisexual Galton-Watson Processes. Journal of Applied Probability, 21 (1984), 915-919.

[6] J. Chalupa, P.L. Leath \& G.R. Reich. Bootstrap percolation on a Bethe latice. J. Phys. C, 12 (1979), L31-L35.

[7] G.R. Grimmett. Percolation, Springer Verlag: New York (1989).

[8] G.R. Grimmett \& I. Manolescu. Inhomogeneous bond percolation on square, triangular, and hexagonal lattices. The Annals of Probability, 41(4) (2013), 2990-3025.

[9] P. Haccou, P. Jagers \& V.A. Vatutin (eds.). Branching Processes: Variation, Growth and Extinction of Populations. Cambridge University Press, Cambridge (2005).

[10] H. Kester. Percolation Theory for Mathematicians, Birkhäuser, Boston (1982).

[11] J. van den Berg, D. Kiss \& P. Nolin. A Percolation process on the binary tree where large finite clusters are frozen. Electronic Communications in Probability, 17(2) (2012), 1-11.

[12] E.E. Vogel, W. Lebrecht \& J.F. Valdés. Bond percolation for homogeneous two-dimensional lattices. Physica A, 389(8) (2010), 1512-1520.

[13] Z. Dogruyol, N. Arsu \& O. Pekcan. Critical exponents of photoinitiated gelation at different light intensities. Journal of Macromolecular Science, Part B: Physics, 48 (2009), 745-754. 